\title{
Nonadherence to antihypertensive medications and associated factors in general medicine clinics
}

\author{
This article was published in the following Dove Press journal: \\ Patient Preference and Adherence \\ I August 2016 \\ Number of times this article has been viewed
}

\section{Mohammed Al Ghobain ${ }^{1,2}$ $\mathrm{H}$ Alhashemi $\mathrm{i}^{1,2}$ \\ A Aljama ${ }^{3}$ \\ S Bin Salih ${ }^{1,2}$ \\ Z Assiri ${ }^{4}$ \\ A Alsomali ${ }^{4}$ \\ Gamal Mohamed ${ }^{5}$ \\ 'Department of Medicine, College of Medicine, King Saud Bin Abdulaziz University for Health Sciences, \\ ${ }^{2}$ King Abdullah International Medical Research Centre, ${ }^{3}$ Department of Medicine, King Abdulaziz Medical City, ${ }^{4}$ College of Nursing, ${ }^{5}$ College of Public Health, King Saud Bin Abdulaziz University for Health Sciences, Riyadh, Kingdom of Saudi Arabia}

Correspondence: Mohammed Al Ghobain Department of Medicine, College of Medicine, King Saud Bin Abdulaziz University for Health Sciences, PO Box 90068, Riyadh II32I, Kingdom of Saudi Arabia Tel/fax +966 I 2520088

Email alanezi@hotmail.com
Objectives: Nonadherence to antihypertensive medications has not been assessed in the Saudi population. The aim of this study was to address and evaluate the magnitude of nonadherence among hypertensive patients and the risk factors associated with it.

Methods: A cross-sectional survey was conducted on hypertensive patients who attended the general internal medicine clinics at King Abdulaziz Medical City, Riyadh, Saudi Arabia, using a questionnaire that was modified after reviewing the literature. Hypertensive patients were labeled as nonadherent if they missed their medications for a total of 7 days during the previous month.

Results: A total of 302 patients participated in the study, of whom $63 \%$ were females with a mean age of 64 years, and $64 \%$ were illiterate. The prevalence of nonadherence to medications among hypertensive patients was found to be $12.3 \%$. Poor disease knowledge was reported in $80 \%$ of patients, while $66 \%$ of the patients had poor monitoring of their disease. Younger age ( $\leq 65$ years), poor monitoring, and uncontrolled blood pressure (BP $\geq 140 / 90 \mathrm{mmHg}$ ) were the predictor factors associated with nonadherence (odds ratio $[\mathrm{OR}]=2.04, P=0.025 ; \mathrm{OR}=2.39$, $P=0.004$; and $\mathrm{OR}=2.86, P=0.003$, respectively).

Conclusion: Nonadherence to antihypertensive medications is lower than that previously reported in the literature. Younger age, uncontrolled BP, and poor monitoring are the main risk factors associated with nonadherence.

Keywords: nonadherence, hypertension, Saudi Arabia, uncontrolled blood pressure

\section{Introduction}

Uncontrolled blood pressure (BP) is defined as BP measurement of $\geq 140 / 90 \mathrm{mmHg}$, which carries high morbidity and mortality risk, including but not limited to heart failure, coronary heart disease, stroke, and renal insufficiency. ${ }^{1,2}$ Nonadherence to antihypertensive medications is a major risk factor that contributes to uncontrolled $\mathrm{BP}$ and subsequently the development of the hypertensive complications., ${ }^{3,4}$ Despite the availability of effective medications, the control of high BP is far below ideal. Hypertensive patients are commonly defined as adherent when they take at least $80 \%$ of their medications on a daily basis. ${ }^{5}$ Rates of nonadherence to medications among hypertensive patients have varied in the literature between $30 \%$ and $50 \%{ }^{6-8}$ This variation is related to multiple factors including level of education, access to medications, and numbers of antihypertensive drugs used by the patients and their side effects.

Although nonadherence to hypertensive medications is a global problem, it has not been investigated previously among hypertensive patients in Saudi Arabia. The magnitude of the problem and factors that contribute to nonadherence to antihypertensive medications are not known in our local population. Therefore, our study aimed to address the magnitude of the problem of nonadherence in hypertensive patients and 
the risk factors associated with such a problem. Moreover, we aimed to evaluate the degree of control of high BP among the hypertensive patient population attending general internal medicine clinics at a single center from Riyadh, Saudi Arabia.

\section{Methods}

A cross-sectional survey was conducted on hypertensive patients who attended the general internal medicine clinics at King Abdulaziz Medical City, Riyadh, Saudi Arabia. King Abdulaziz Medical City is a tertiary care and academic center with a total of 800 beds affiliated with King Saudi Ben Abdulaziz University for health sciences. The total number of internal medicine clinics are 16 clinics/wk that are run by 12 certified internists; patients attending the clinics are usually Saudi Arabian citizens referred from primary care clinics.

The survey questionnaire was constructed after reviewing the literature on the topic. Items included in the questionnaire were age, sex, complexity of therapy, side effects, use of other medications, comorbid conditions, medications' administration, smoking, disease knowledge, BP monitoring, socioeconomic status, BP control, and education level. Complex antihypertensive therapy was defined as needing more than two medications to control the BP with a frequency of more than once per day. ${ }^{3}$ Good knowledge was defined as knowing the target BP and two complications of high BP, while good monitoring was defined as measuring the BP at least once monthly by the patient or health care facility. ${ }^{9}$ Initially, the questionnaire was used as pilot on ten patients, and then it was discussed with the study personnel and the clinic staff. Using the feedback from patients, study personnel, and the clinic staff, the survey questionnaire was assessed for content validity and modified to its final version. The target population of the survey was adult hypertensive patients attending the general internal medicine clinic during the month of April 2012. The sample used was all the hypertensive patients who attended the general medicine clinic during the month of April 2012. The primary investigator trained a team of three health workers on using the survey form for data collection to ensure uniformity of data collection.

Patients who were eligible to participate in the survey were those who had been diagnosed with hypertension for $>6$ months and were 18 years or older. Clinical and pharmacy records for all general medicine clinic patients were screened every morning by the clinic staff. Any patient whose clinical and pharmacy records indicated that he/she had hypertension for $\geq 6$ months was then approached by the survey team. Patients who were pregnant and breast-feeding, patients with dementia, or those who refused to participate in the survey were excluded. Hypertensive patients are defined as adherent when they take at least $80 \%$ of their medications on a daily basis. This definition was changed to the number of days to make it easier to understand for illiterate patients; hypertensive patients were labeled as nonadherent if they missed their medications for a total of 7 days during the previous month. Patients' BP was considered uncontrolled if the BP measurement in the clinic was $\geq 140 / 90 \mathrm{mmHg}$.

The BP was measured by the general medicine clinic nurses using the automated BP machine available in the outpatient clinics (Datascope Accutorr Plus). It was validated as a tool for measuring BP after being tested against mercury sphygmomanometer. ${ }^{10,11}$ The BP reading was taken after a period of rest and measured in the seating position. If the systolic BP was $\geq 140 \mathrm{mmHg}$ or the diastolic BP $\geq 90 \mathrm{mmHg}$, the nursing staff would double-check the fit of the BP cuff, repeat the measurement after 5 minutes, and record the second BP measurement in the chart. The study protocol was approved by the Ethical Research Committee of King Abdullah International Medical Research Center.

\section{Sample size and statistical analysis}

General internal medicine clinics were 16 clinics/wk with an average of ten patients per clinic (160 patients/wk). This adds up to $\sim 8,000$ patient-visits per year for all medical problems, as the target population for the survey. Assuming that the prevalence of nonadherence was 30\% and accepting $5 \%$ margin of error with $95 \%$ confidence interval (CI) and a population size of 8,000 , the required sample size was estimated to be 302 patients. The sample size estimation was performed using Epi-info, while SPSS (Version 21; IBM Corporation, Armonk, NY, USA) was used for data entry and data analysis. The nonadherence/adherence binary variable was treated as the outcome variable and was assessed against the rest of the items of the survey questionnaire as possible explanatory variables using logistic regression. The prevalence of nonadherence in the target population was estimated, and the significant risk factors associated with it were identified using multiple logistic regression. In addition, the proportion of patients with controlled BP was estimated along with the average of their BP level as a quality measure for the performance of the general medicine clinics.

\section{Results}

A total of 302 patients signed written informed consent and participated in the study out of 654 patients who visited the clinics (participation rate: $46 \%$ ), of whom $63 \%$ were females with a mean age of 64 years, and $64 \%$ were illiterate 
(did not receive formal education). The prevalence of nonadherence to medications among hypertensive patients was found to be $12.3 \%$ in surveyed sample, $57 \%$ of them were using complex regimen therapy, and $62 \%$ did not report side effects. Poor disease knowledge was reported in $80 \%$ of patients, while $66 \%$ of the patients had poor monitoring of their disease. The most prevalent comorbidities affecting hypertensive patients were hypercholesterolemia and diabetes, with prevalence rates of $83 \%$ and $73 \%$, respectively. Ischemic heart disease and cerebrovascular disease affected $14 \%$ and $9 \%$ of the patients, respectively, while $13 \%$ of the patients had chronic renal failure and $12 \%$ had congestive heart failure.

Logistic regression analysis was performed to predict factors that may increase the risk of nonadherence. Younger age ( $\leq 65$ years), poor monitoring, and uncontrolled $\mathrm{BP} \geq 140 / 90 \mathrm{mmHg}$ were the predictor factors associated with nonadherence (odds ratio $[\mathrm{OR}]=2.04, P=0.025$; OR $=2.39$, $P=0.004$; and $\mathrm{OR}=2.86, P=0.003$, respectively; Table 1).

The survey showed that 189 patients $(63 \%)$ had controlled BP $(<140 / 90 \mathrm{mmHg})$, while 113 patients $(37 \%)$ had uncontrolled BP. The mean BP measurement for the patients with controlled BP was 123/68 mmHg. On the other hand, the mean BP measurement for the patients with uncontrolled $\mathrm{BP}$ was $153 / 77 \mathrm{mmHg}$, with an estimated 95\% CI ranging from $156 / 79 \mathrm{mmHg}$ to $152 / 74 \mathrm{mmHg}$.

\section{Discussion}

Our study showed that $12.3 \%$ of hypertensive patients in our center were nonadherent to antihypertensive medications. Among all patients, 57\% of them were using complex regimen therapy and $62 \%$ did not report side effects. Poor disease knowledge was reported in $80 \%$ of patients, while $66 \%$ of the patients had poor monitoring of their disease and $63 \%$ of the patients had controlled BP. Factors that may increase the risk of nonadherence were younger age, poor monitoring, and uncontrolled BP.

To our knowledge, this is the first study addressing the magnitude of nonadherence to antihypertensive medications and estimating the risk of nonadherence in patients with uncontrolled BP in Saudi Arabia however, many clinicians observed, in their daily practice, an increase in the number of patients who were not adherent especially when BP was not well controlled.

Monitoring patients' adherence is not an easy task. Monitoring procedures range from simple methods such as self-reporting and pill count to more complex methods such as drug assays and microelectronic monitors. Since all the monitoring procedures have their limitations, self-reporting was used to estimate nonadherence. ${ }^{12}$ Self-reporting was more pragmatic; however, it carried an unavoidable risk of recall bias. It is likely that patients' memory recall led to underreporting of nonadherence and subsequently to the low nonadherence estimate in this survey. The impact of recall bias on the estimated nonadherence can be predicted from previous literature. In a previous study, self-reporting resulted in $17 \%$ overestimation of patients' compliance in comparison to actual pill count. ${ }^{13}$

In comparison to our study of self-reported nonadherence, with administrative database studies of combined nonadherence and nonpersistence, a nonadherence prevalence of $12 \%$ is still considered to be low. ${ }^{6-8}$ However, Mc Namara et al ${ }^{14}$ reported $15 \%$ nonadherence rate to medication, and Vrijens et a ${ }^{15}$ reported that about half of the patients who were prescribed an antihypertensive drug had stopped taking it within 1 year.

Poor knowledge about the disease and the lack of BP monitoring were universally high across the whole survey sample; $80.5 \%$ and $62 \%$, respectively. Both the poor knowledge and lack of monitoring can be explained by the high illiteracy rate found in the sample. The fact that $66 \%$ of the patients were not able to read or write is an important characteristic of the target population that deserves special attention when designing health education material, to improve the knowledge of the patients in the future.

Uncontrolled BP ( $\geq 140 / 90 \mathrm{mmHg}$ ) and younger age ( $\leq 65$ years) were significant risk factors associated with nonadherence, $\mathrm{OR}=3$ and $\mathrm{OR}=2.6$, respectively.

Morgado et $\mathrm{al}^{9}$ reported the predictors of medication associated with nonadherence to be: unawareness of target BP values, a report of drug's side effects, lack of BP monitoring, and unawareness of medication indications.

Therefore, treating physicians have to rule out nonadherence before increasing the dosage of antihypertensive medications in patients with uncontrolled BP. This can be performed through proper communication, education, and feedback from the patient. Increasing the number of medications or dosage for nonadherent patients will increase the risk of developing side effects and subsequently promote further nonadherence. ${ }^{12}$

There are many other reasons why BP was uncontrolled apart from nonadherence to medications such as white coat hypertension, resistant hypertension, and patients with suboptimal treatment. According to the US data, approximately one-third of the patients with uncontrolled BP have white coat hypertension, and $20 \%$ of the patients with uncontrolled BP have resistant hypertension. ${ }^{16,17}$ 
Table I Baseline characteristics of the study population and estimated ORs between the nonadherence and adherence group in relation to possible explanatory variables using logistic regression analysis

\begin{tabular}{|c|c|c|c|c|c|c|}
\hline Variables & $\begin{array}{l}\text { Total study population: } \\
N=302 \text { (\%) }\end{array}$ & $\begin{array}{l}\text { Nonadherence: } \\
n=\mathbf{3 7}(\%)\end{array}$ & $\begin{array}{l}\text { Adherence: } \\
\mathrm{n}=265\end{array}$ & OR & $95 \% \mathrm{Cl}$ & $P$-value \\
\hline \multicolumn{7}{|l|}{ Sex } \\
\hline$M$ & 112 (37) & $16(14)$ & 96 & 1.34 & $0.67-2.7$ & 0.40 \\
\hline $\mathrm{F}$ & $190(63)$ & $21(I I)$ & 169 & & & \\
\hline \multicolumn{7}{|l|}{ Age, years } \\
\hline$\leq 65$ & $159(53)$ & $27(17)$ & 132 & 2.04 & $0.98-4.22$ & 0.025 \\
\hline$>65$ & $143(47)$ & $14(9)$ & 129 & & & \\
\hline \multicolumn{7}{|c|}{ Complex therapy } \\
\hline Yes & $|7|(57)$ & $17(10)$ & 154 & 0.61 & $0.30-1.22$ & 0.16 \\
\hline No & $|3|(43)$ & $20(15)$ & III & & & \\
\hline \multicolumn{7}{|l|}{ Side effects } \\
\hline Yes & II4 (38) & $14(12)$ & 100 & 1.00 & $0.49-2.04$ & 0.99 \\
\hline No & $179(62)$ & $23(13)$ & 156 & & & \\
\hline \multicolumn{7}{|c|}{ Poor disease knowledge } \\
\hline Yes & $243(80)$ & $30(12)$ & 213 & 1.04 & $0.43-2.51$ & 0.91 \\
\hline No & $59(20)$ & $7(I I)$ & 52 & & & \\
\hline \multicolumn{7}{|c|}{ Comorbid conditions } \\
\hline Yes & $287(95)$ & $35(12)$ & 252 & 0.90 & $0.19-4.17$ & 0.89 \\
\hline No & $15(5)$ & $2(13)$ & 13 & & & \\
\hline \multicolumn{7}{|c|}{ Current smoker } \\
\hline Yes & II (4) & I (9) & 10 & 0.71 & $0.09-5.69$ & 0.74 \\
\hline No & $291(96)$ & $36(12)$ & 255 & & & \\
\hline \multicolumn{7}{|c|}{ Poor monitoring } \\
\hline Yes & $200(66)$ & $30(15)$ & 170 & 2.39 & $\mathrm{I} .0 \mathrm{I}-5.66$ & 0.040 \\
\hline No & $102(34)$ & $7(7)$ & 95 & & & \\
\hline \multicolumn{7}{|c|}{ Illiterate patients } \\
\hline Yes & $194(64)$ & $24(12)$ & 170 & 1.01 & $0.50-2.0$ & 0.98 \\
\hline No & $106(36)$ & $13(12)$ & 93 & & & \\
\hline \multicolumn{7}{|c|}{$\mathrm{BP} \geq \mathrm{I} 40 / 90 \mathrm{mmHg}$} \\
\hline Yes & $113(37)$ & $23(20)$ & 90 & 2.86 & $1.40-5.80$ & 0.003 \\
\hline No & $189(63)$ & $15(8)$ & 174 & & & \\
\hline \multicolumn{7}{|l|}{ IHD } \\
\hline Yes & $4 \mid(\mid 4)$ & $3(7)$ & 38 & 0.53 & $0.15-1.80$ & 0.30 \\
\hline No & $261(86)$ & $34(13)$ & 227 & & & \\
\hline \multicolumn{7}{|l|}{ CVA } \\
\hline Yes & $27(9)$ & $4(15)$ & 23 & 1.27 & $0.4|-3.9|$ & 0.67 \\
\hline No & $257(91)$ & $33(13)$ & 242 & & & \\
\hline \multicolumn{7}{|l|}{ CRF } \\
\hline Yes & $39(13)$ & $5(13)$ & 34 & 1.06 & $0.38-2.91$ & 0.90 \\
\hline No & $263(87)$ & $32(12)$ & 231 & & & \\
\hline \multicolumn{7}{|l|}{$\mathrm{CHF}$} \\
\hline Yes & $35(12)$ & $3(9)$ & 32 & 0.64 & $0.18-2.21$ & 0.48 \\
\hline No & $265(88)$ & $34(13)$ & 233 & & & \\
\hline \multicolumn{7}{|c|}{ High cholesterol } \\
\hline Yes & $251(83)$ & $33(13)$ & 218 & 1.78 & $0.60-5.26$ & 0.30 \\
\hline No & $51(17)$ & $4(15)$ & 47 & & & \\
\hline \multicolumn{7}{|l|}{ Diabetes } \\
\hline Yes & $222(73)$ & $28(13)$ & 194 & 1.13 & $0.5 \mathrm{I}-2.53$ & 0.75 \\
\hline No & $80(27)$ & $9(11)$ & 71 & & & \\
\hline
\end{tabular}

Abbreviations: $\mathrm{BP}$, blood pressure; $\mathrm{CHF}$, congestive heart failure; $\mathrm{Cl}$, confidence interval; $\mathrm{CRF}$, chronic renal failure; CVA, cerebral vascular accident; $\mathrm{F}$, female; IHD, ischemic heart disease; $M$, male; OR, odds ratio.

To find exactly the proportions of patients with white coat hypertension, resistant hypertension, and undertreated hypertension, patients needed to have ambulatory BP monitoring and documentation of the medications used, ${ }^{18}$ both of which were outside the scope of this study.

The proportion of hypertensive patients with controlled BP (189 patients) in this survey (63\%, with a $95 \%$ CI from
$58 \%$ to $68 \%$ ) is higher than the previously published national figures which ranged from $30 \%$ to $50 \%$ only. ${ }^{19-21}$ It is likely that the proportion of uncontrolled hypertensive patients was underestimated, because this survey was not designed to identify patients with white coat hypertension. The importance of the low nonadherence and the high control of BP identified in the survey cannot be overemphasized, since both are 
associated with better prevention of hypertensive complications and improved morbidity and mortality. Our study has the limitation of being a cross-sectional study with possible biased reporting, in which we used a face-to-face structural questionnaire as a measurement tool for nonadherence.

\section{Conclusion}

Nonadherence to antihypertensive medications in our study is lower than that previously reported in the literature. Younger age, uncontrolled BP, and poor monitoring are the main risk factors for nonadherence.

\section{Disclosure}

The authors report no conflicts of interest in this work.

\section{References}

1. Chobanian AV, Bakris GL, Black HR, et al; Joint National Committee on Prevention, Detection, Evaluation, and Treatment of High Blood Pressure. National Heart, Lung, and Blood Institute, National High Blood Pressure Education Program Coordinating Committee. Seventh report of the joint national committee on prevention detection, evaluation, and treatment of high blood pressure. Hypertension. 2003;42(6): 1206-1252.

2. Handler J, Lackland DT. Translation of hypertension treatment guidelines into practice: a review of implementation. J Am Soc Hypertens. 2011; 5(4):197-207.

3. Munger MA, Van Tassel BW, LaFleur J. Medication nonadherence: an unrecognized cardiovascular risk factor. MedGenMed. 2007;9(3):58.

4. Herttua K, Tabák AG, Martikainen P, Vahtera J, Kivimäki M. Adherence to antihypertensive therapy prior to the first presentation of stroke in hypertensive adults: population-based study. Eur Heart J. 2013;34(38): 2933-2939.

5. Nair KV, Belletti DA, Doyle JJ, et al. Understanding barriers to medication adherence in the hypertensive population by evaluating responses to a telephone survey. Patient Prefer Adherence. 2011; 5:195-206.

6. Psaty BM, Koepsell TD, Yanez ND, et al. Temporal patterns of antihypertensive medication use among older adults. 1989 through 1992: an effect of the major clinical trials on clinical practice? JAMA. 1995; 273(18):1436-1438.
7. Caro JJ, Speckman JL, Salas M, Raggio G, Jackson JD. Effect of initial drug choice on persistence with antihypertensive therapy: the importance of actual practice data. CMAJ. 1999;160(1):41-46.

8. Schroeder K, Fahey T, Ebrahim S. How can we improve adherence to blood pressure-lowering medication in ambulatory care? Systematic review of randomized controlled trials. Arch Intern Med. 2004; 164(7):722-732.

9. Morgado M, Rolo S, Macedo AF, Pereira L, Castelo-Branco M. Predictors of uncontrolled hypertension and antihypertensive medication nonadherence. J Cardiovasc Dis Res. 2010;1(4):196-202.

10. Wong SN, Tz Sung RY, Leung LC. Validation of three oscillometric blood pressure devices against auscultatory mercury sphygmomanometer in children. Blood Press Monit. 2006;11(5):281-291.

11. Khawaja RA, Qureshi R, Mansure AH, Yahya ME. Validation of Datascope Accutorr PlusTM using British Hypertension Society (BHS) and Association for the Advancement of Medical Instrumentation (AAMI) protocol guidelines. J Saudi Heart Assoc. 2010;22(1):1-5.

12. Wuerzner K, Hassler C, Burnier M. Difficult blood pressure control: watch out for non-compliance! Nephrol Dial Transplant. 2003;18(10): 1969-1973.

13. Haynes RB, Taylor DW, Sackett DL, Gibson ES, Bernholz CD, Mukherjee J. Can simple clinical measurements detect patient noncompliance? Hypertension. 1980;2(6):757-764.

14. Mc Namara KP, Versace VL, Marriott JL, Dunbar JA. Patient engagement strategies used for hypertension and their influence on selfmanagement attributes. Fam Pract. 2014;31(4):437-444.

15. Vrijens B, Vincze G, Kristanto P, Urquhart J, Burnier M. Adherence to prescribed antihypertensive drug treatments: longitudinal study of electronically compiled dosing histories. BMJ. 2008;336(7653):1114-1117.

16. de la Sierra A, Segura J, Banegas JR, et al. Clinical features of 8295 patients with resistant hypertension classified on the basis of ambulatory blood pressure monitoring. Hypertension. 2011;57(5):898-902.

17. Roberie DR, Elliott WJ. What is the prevalence of resistant hypertension in the United States? Curr Opin Cardiol. 2012;27(4):386-391.

18. Sarafidis PA, Georgianos P, Bakris GL. Resistant hypertension - its identification and epidemiology. Nat Rev Nephrol. 2013;9(1):51-58.

19. Al-Khaldi YM. Quality of hypertension care in the family practice center, Aseer Region, Saudi Arabia. J Family Community Med. 2011;18(2): $45-48$.

20. Saeed AA, Al-Hamdan NA, Bahnassy AA, Abdalla AM, Abbas MA, Abuzaid LZ. Prevalence, awareness, treatment, and control of hypertension among Saudi adult population: a national survey. Int $J$ Hypertens. 2011;2011:174135.

21. Al-Tuwijri AA, Al-Rukban MO. Hypertension control and comorbidities in primary centers in Riyadh. Ann Saudi Med. 2006;26(4):266-271.
Patient Preference and Adherence

\section{Publish your work in this journal}

Patient Preference and Adherence is an international, peer-reviewed, open access journal that focuses on the growing importance of patient preference and adherence throughout the therapeutic continuum. Patient satisfaction, acceptability, quality of life, compliance, persistence and their role in developing new therapeutic modalities and compounds to optimize

\section{Dovepress}

clinical outcomes for existing disease states are major areas of interest for the journal. This journal has been accepted for indexing on PubMed Central The manuscript management system is completely online and includes a very quick and fair peer-review system, which is all easy to use. Visit http://www. dovepress.com/testimonials.php to read real quotes from published authors. 